\title{
Microcystic Corneal Edema
}

National Cancer Institute

\section{Source}

National Cancer Institute. Microcystic Corneal Edema. NCI Thesaurus. Code C50901.

Corneal edema of inflammatory orig in associated with contact lens wear and morphologically characterized by presence of epithelial microcysts. Microcysts are small (typically 10-50 microns in diameter), clear, irregularly shaped high refractive inclusions that form in the basal layers of the epithelium and move towards the anterior surface of the cornea, surrounding epithelial haze. The cause of microcytic edema is related to the physical presence of contact lenses and possibly a mechanical effect of lens wear. 